\title{
Visual loss from retinal oedema in autosomal dominant exudative vitreoretinopathy
}

\author{
D. SWANSON, P. RUSH, AND A. C. BIRD
}

From Moorfields Eye Hospital, City Road, London EC1V 2PD

SUMMARY We examined 3 family members with autosomal dominant exudative vitreoretinopathy. The propositus was unusual in that she noticed reduced vision in her second eye at the age of 23 due to retinal oedema. Her mother and sister were asymptomatic, but fluorescein angiography demonstrated phenotypic expression of the abnormal gene.

Autosomal dominant exudative vitreoretinopathy was first described by Criswick and Schepens ${ }^{1}$ as a bilateral, familial, progressive disease simulating retrolental fibroplasia but with no history of prematurity or oxygen administration. Gow and Oliver $^{2}$ later showed autosomal dominant inheritance of this condition. Ober et al. ${ }^{3}$ confirmed the inherit-

Correspondence to Professor A. C. Bird. ance pattern and delineated the spectrum of its expression more fully. They emphasised that the major threats to vision are haemorrhage and retinal detachment, that progression is limited to the early years of life, and specifically that visual loss has not been recorded in patients older than 18 years. In this report we present a patient with autosomal dominant exudative vitreoretinopathy who lost vision at a later age and from different causes than previously reported.

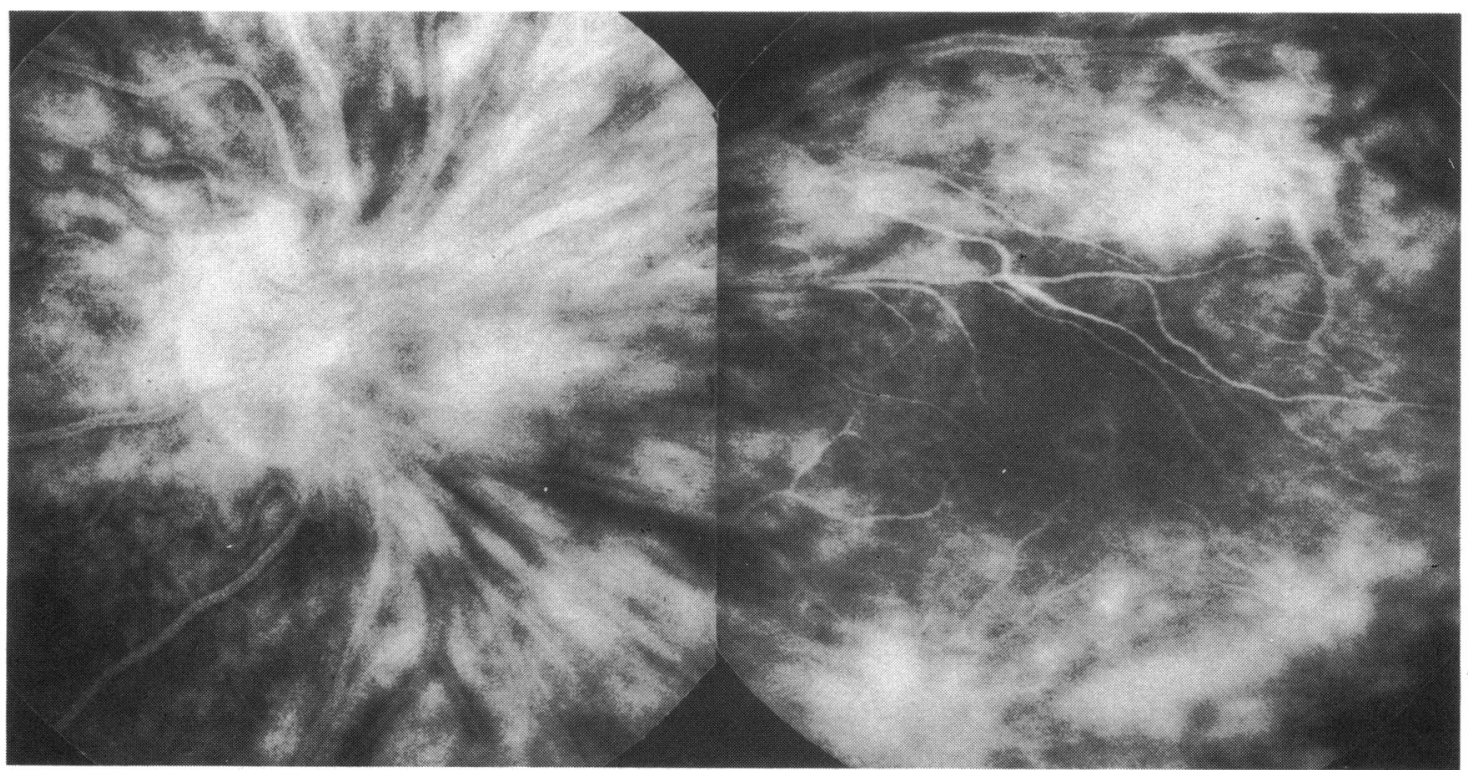

Fig. 1a

Fig. 1 Posterior retinal oedema and distortion of the retinal vessels in the left eye with macular oedema (1a) and peripheral vascular leakage (1b). 


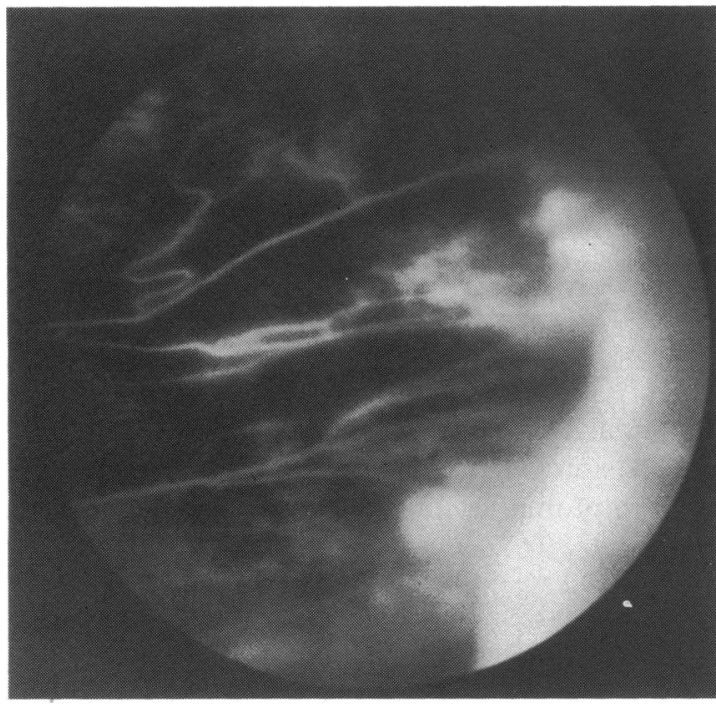

Fig. 1b

\section{Case report}

A white girl was first seen in 1961 at the age of 5 years with a 2-year history of squint. She was born at term with birth weight of $6.75 \mathrm{lb}(3065 \mathrm{~g})$ and had not received oxygen therapy. Her vision was counting fingers with the right eye and 6/9 with the left. A retinal detachment was found in the right eye and distortion of the retinal vessels was noted in the left eye. A tentative diagnosis of von Hippel's disease was made, and no treatment was undertaken.

She was seen again in August 1980 at the age of 23 years with a 2-month history of blurred vision in the left eye. At that time her vision was $6 / 24$ with correction $(-3.00 \mathrm{D}$ sph/-1.50 D cyl $\times 80 \mathrm{deg})$. Examination and fluorescein angiography demonstrated closure and straightening of the retinal vessels in the periphery, but the most significant abnormalities were widespread oedema affecting the posterior retina and distortion of the retinal blood vessels (Fig. 1). The peripheral vascular changes suggested the diagnosis of autosomal dominant exudative vitreoretinopathy, and that was confirmed when angiography revealed characteristic closure and leakage in the peripheral retinas of both her mother and sister (Figs. 2, 3). They too had been born at term and were of normal birth weight, $7 \cdot 5$ and $6.0 \mathrm{lb}(3405$ and $2724 \mathrm{~g}$ ) respectively. Both are asymptomatic and appear normal ophthalmoscopically. No other affected members were identified by history alone (Fig. 4).

\section{Discussion}

Recently Ober et al. ${ }^{3}$ reviewed 3 pedigrees of autosomal dominant exudative vitreoretinopathy with 12 members having identifiable clinical involvement. They concluded from observations of their own patients and from a review of the literature that many patients who possess the abnormal gene may remain permanently asymptomatic despite fluoroscopic

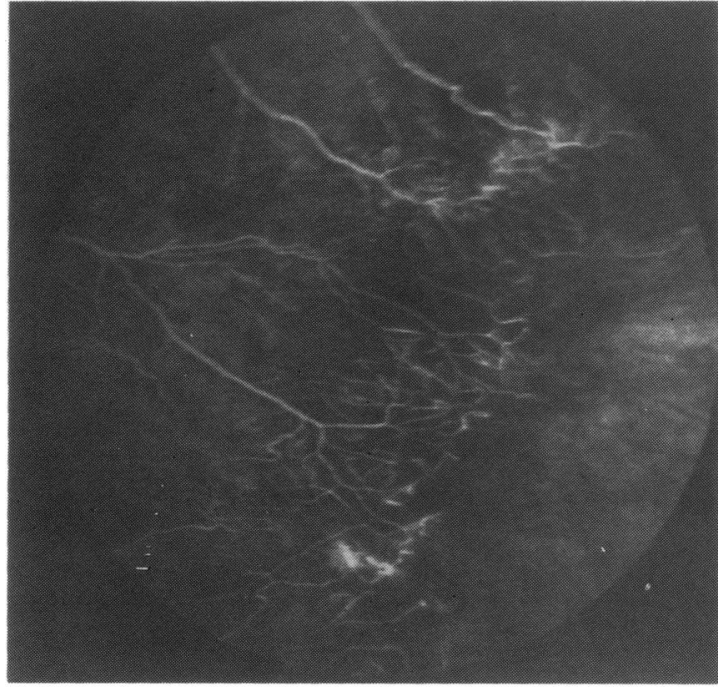

Fig. 2a

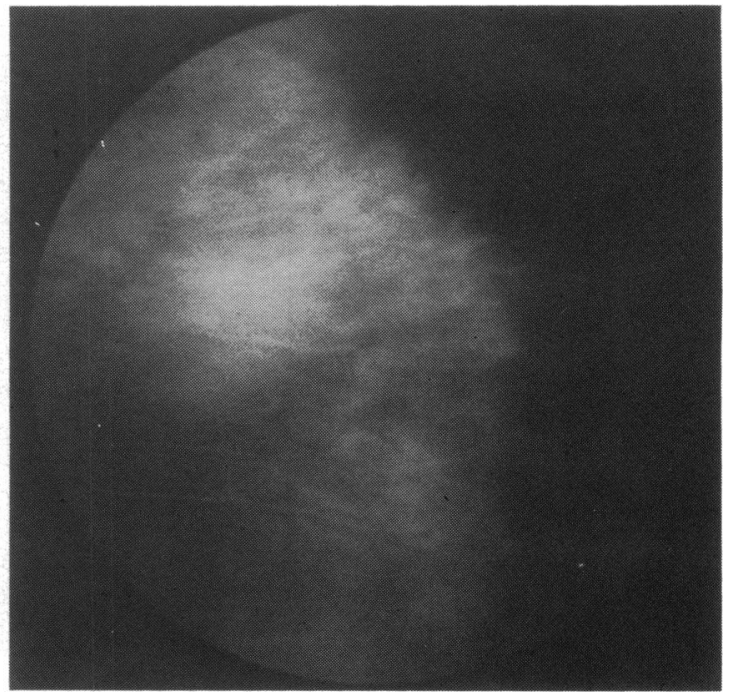

Fig. 2b

Fig. 2a, b Abnormal peripheral vessels and leakage in left eye of patient's mother. 


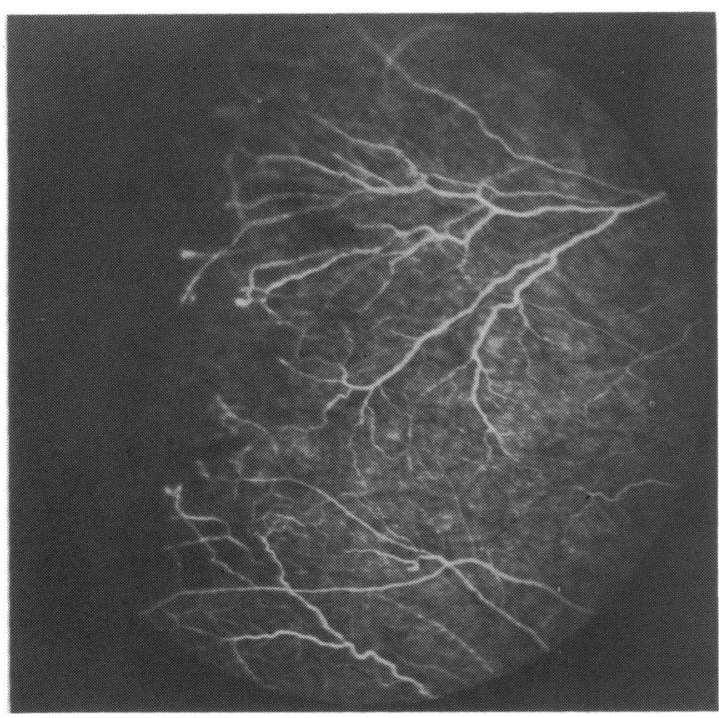

Fig. 3 Abnormal peripheral vessels in right eye of patient's sister.

evidence of abnormal vascular closure and permeability, that the severity is not clearly correlated with age, and that the onset of visual loss has not been recorded after the age of 18 . Our experience with the family described in this report supports in part their conclusions. In particular the diagnosis of inherited disease was identified only after examination of potentially affected relatives.

The propositus differed from patients previously reported in 2 significant aspects. First, at age 23 years she is the oldest patient to have experienced visual loss. Secondly, retinal oedema has not been recorded as a cause of visual loss in this disorder. Although retinal oedema has been described and its relationship to retinal vascular leakage illustrated, ${ }^{3}$ our patient is unusual in that the amount and extent of oedema was out of proportion to the degree of retinal traction. This observation suggests that the oedema is not due solely to distortion of the retinal blood vessels and may imply an intrinsic vascular abnormality giving rise to abnormal vascular permeability.

We know of no prophylactic or therapeutic procedure which would have altered the course of our patient's condition, and we agree with the suggestion ${ }^{3}$ that the need for prophylactic intervention is doubtful.

\section{References}

1 Criswick VG, Schepens CL. Familial exudative vitreoretinopathy. Am J Ophthalmol 1969; 68: 578-94.

2 Gow CLB, Oliver GL. Familial exudative vitreoretinopathy: an expanded view. Arch Ophthalmol 1971; 86: 150-5.

3 Ober RR, Bird AC, Hamilton AM, Sehmi K. Autosomal dominant exudative vitreoretinopathy. Br J Ophthalmol 1980; 64: 112-20.

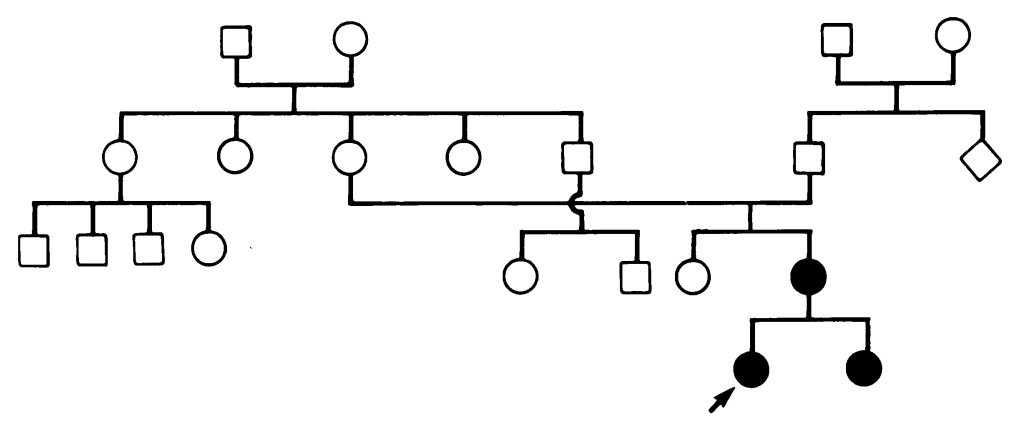

Fig. 4 Pedigree. 